\title{
A Phase 1 Pharmacokinetic Study of Cysteamine Bitartrate Delayed-Release Capsules Following Oral Administration with Orange Juice, Water, or Omeprazole in Cystinosis
}

\author{
Danielle Armas • Robert J. Holt • Nils F. Confer • Gregg C. Checani • \\ Mohammad Obaidi · Yuli Xie · Meg Brannagan
}

Received: November 15, 2017 / Published online: February 6, 2018

(C) The Author(s) 2018. This article is an open access publication

\begin{abstract}
Introduction: Cystinosis is a rare, metabolic, autosomal recessive, genetic lysosomal storage disorder characterized by an accumulation of cystine in various organs and tissues. Cysteamine bitartrate (CB) is a cystine-depleting aminothiol agent approved in the United States and Europe in immediate-release and delayedrelease (DR) formulations for the treatment of nephropathic cystinosis in children and adults. It is recommended that CBDR be administered with fruit juice (except grapefruit juice) for maximum absorption. Omeprazole is a proton pump inhibitor that inhibits gastric acid
\end{abstract}

Enhanced content To view enhanced content for this article go to http://www.medengine.com/Redeem/ C6629960531EE908.

D. Armas

Celerion Inc., Phoenix, AZ, USA

R. J. Holt · N. F. Confer · G. C. Checani

Horizon Pharma, Inc., Lake Forest, IL, USA

R. J. Holt

College of Pharmacy, University of Illinois-Chicago,

Chicago, IL, USA

M. Obaidi $(\square)$

Clinical Pharmacology and Pharmacometrics,

Celerion Inc., Lincoln, NE, USA

e-mail: Mohammadullah.obaidi@celerion.com

Y. Xie $\cdot$ M. Brannagan

Celerion Inc., Lincoln, NE, USA secretion and, theoretically, may cause the premature release of cysteamine by increasing intragastric $\mathrm{pH}$, thereby affecting the $\mathrm{PK}$ of CBDR.

Methods: This open-label, three-period, randomized study in healthy adult subjects was designed primarily to compare the pharmacokinetics of CBDR capsules after a single oral dose administered with orange juice, water, or multiple oral doses of omeprazole with water at steady state. A total of 32 subjects were randomly assigned to receive study agents in one of two treatment sequences.

Results: All subjects completed the study and baseline characteristics of the overall population and the two treatment sequence populations were similar. Peak mean plasma cysteamine concentrations following co-administration of CBDR capsules with orange juice $(1892 \mathrm{ng} / \mathrm{mL})$ were higher compared with co-administration with water (1663 ng/mL) or omeprazole $20 \mathrm{mg}$ and water $(1712 \mathrm{ng} / \mathrm{mL})$. Mean time to peak plasma concentration was shorter with omeprazole coadministration $(2.5 \mathrm{~h})$ compared with orange juice $(3.5 \mathrm{~h})$ or water $(3.0 \mathrm{~h})$. Statistical comparisons between treatment groups indicated that exposure as assessed by $\mathrm{AUC}_{0-t}, \mathrm{AUC}_{0-\infty}$, and $C_{\max }$ were all within the $80-125 \%$ bioequivalence ranges for all comparisons. All treatments were generally well tolerated.

Conclusion: Overall, the pharmacokinetics of cysteamine bitartrate DR capsules are not 
significantly impacted by co-administration with orange juice, water only, or omeprazole (with water).

Funding: Horizon Pharma, Inc.

Keywords: Cysteamine bitartrate; Cystinosis; Omeprazole; Pharmacokinetics; Proton pump inhibitor

\section{INTRODUCTION}

Cystinosis is a rare, metabolic, autosomal recessive, genetic lysosomal storage disorder characterized by an accumulation of cystine in various organs and tissues [1, 2]. Infantile nephropathic cystinosis is the most common (95\% of cases) and severe form of the disease, and is associated with progressive renal failure that can lead to end-stage renal disease by midchildhood if left untreated [1].

Cysteamine bitartrate is a cystine-depleting aminothiol agent approved in the United States and Europe in immediate-release and delayedrelease (DR) formulations for the treatment of nephropathic cystinosis in children and adults $[3,4]$. Cysteamine bitartrate DR therapy taken consistently at regular intervals has been shown to reduce leukocyte cystine levels, the rate of decline in renal function, and progression to renal failure if initiated early [2, 5-7].

Cysteamine bitartrate DR capsules (Procysbi $^{\circledR}$; Horizon Pharma, Lake Forest, IL, USA) are an enteric-coated formulation designed to prevent premature release of cysteamine in the stomach and thereby increase bioavailability by allowing absorption from the duodenum, where gastrointestinal $\mathrm{pH}$ is higher. Studies have shown that cysteamine bitartrate DR is noninferior to the immediate-release formulation in terms of maintaining low leukocyte cystine levels but at a lower total daily dose and reduced dosing frequency $[8,9]$. Cystine levels rise very quickly when therapy is interrupted, and patients frequently have difficulty with adherence because of the drug's potential to cause gastrointestinal irritation. Delaying and sustaining absorption of cysteamine may, therefore, have a beneficial effect on patient adherence, which is a recognized issue with cysteamine therapy [2].

A previous pharmacokinetic (PK) study determined that optimal absorption of cysteamine bitartrate DR capsules occurs during the fasting state ( $>30$ min before a carbohydrate-rich meal) [10]. It is also recommended that cysteamine bitartrate DR be administered with fruit juice (except grapefruit juice) for maximum absorption [4]. Omeprazole is a proton pump inhibitor that inhibits gastric acid secretion and, theoretically, may cause the premature release of cysteamine by increasing intragastric $\mathrm{pH}$, thereby affecting the $\mathrm{PK}$ of cysteamine bitartrate. A pilot PK evaluation in 20 healthy subjects demonstrated that concomitant omeprazole administration did not significantly affect the primary PK parameters of cysteamine bitartrate DR (data not shown).

As part of a post-marketing commitment to support the new drug application and to inform administration methods, the current study was designed to assess the effects of multiple-dose omeprazole and water or orange juice on cysteamine PK and bioavailability. Specifically, the primary objectives of this study were to compare the key PK parameters of cysteamine bitartrate DR capsules administered with (1) orange juice or (2) water, as well as with (3) multiple oral doses of omeprazole with water, such that omeprazole was at steady state. This study design will confirm whether multipledose omeprazole affects the PK and bioavailability of cysteamine when cysteamine bitartrate DR is administered with water instead of orange juice.

\section{METHODS}

\section{Study Design}

All procedures performed in studies involving human participants were in accordance with the ethical standards of the institutional and/or national research committee and with the 1964 Helsinki declaration and its later amendments or comparable ethical standards. Informed consent was obtained from all individual participants included in the study. Approval for 
this study was granted by Chesapeake Research Review.

This was an open-label, single-dose, threeperiod, randomized study in healthy adult subjects. Cysteamine bitartrate DR capsules (Procysbi ${ }^{\circledR}$; Horizon Pharma, Inc., Lake Forest, Illinois, USA) were supplied as 75-mg capsules. Omeprazole was supplied as 20-mg delayedrelease capsules (Mylan Pharmaceuticals, Canonsburg, PA, USA). A single branded orange juice (SUNCUP ${ }^{\mathrm{TM}}$ ) was used for all subjects. Any PK effects on cysteamine from PPIs would be expected to be the same with similar acting drugs with similar effects on gastric $\mathrm{pH}$; therefore, no other PPIs were studied. All study drugs were administered orally following an overnight fast, and subjects were instructed not to crush, split, or chew the capsules.

\section{Study Population}

Healthy adult nonsmokers, including males and females of non-childbearing potential, aged 18-55 years, with a body mass index (BMI) from 18.5 to $32.0 \mathrm{~kg} / \mathrm{m}^{2}$ at screening, were considered suitable for inclusion. Key exclusion criteria included clinically significant medical/ psychiatric conditions or illnesses that might have confounded the results of the study or posed an additional risk to the subject.

Subjects were free to withdraw from the study at any time for any reason, or may have been withdrawn from the study by the principal or subinvestigator for adverse events (AEs), difficulties in blood collection, or protocol violation.

\section{Treatment Protocol}

Subjects were randomly assigned to receive all of the following treatments $(\mathrm{A}, \mathrm{B}$, and $\mathrm{C})$ in one of two sequences over three periods (ABC, BAC; Fig. 1). Subjects received each treatment on one occasion:

- Treatment A: Cysteamine bitartrate $600 \mathrm{mg}$ $(8 \times 75$-mg DR capsules) at hour 0 on day 1 with approximately $240 \mathrm{~mL}$ orange juice
- Treatment B: Cysteamine bitartrate $600 \mathrm{mg}$ $(8 \times 75-\mathrm{mg}$ DR capsules) at hour 0 on day 1 with approximately $240 \mathrm{~mL}$ water

- Treatment C: Omeprazole $20 \mathrm{mg}$ with approximately $240 \mathrm{~mL}$ water every $24 \mathrm{~h}$ for 5 consecutive days (within $\pm 1 \mathrm{~h}$ of dosing time on day 1), co-administered with cysteamine bitartrate $600 \mathrm{mg}$ at hour 0 on day 5 with approximately $240 \mathrm{~mL}$ water.

Additional water and/or orange juice, up to a maximum of $50 \mathrm{~mL}$, was administered if needed by the subject, with capsule dosing completed within 2 min of the scheduled time.

The 600-mg oral dose of cysteamine bitartrate DR was selected for this study as this is the same dose used in previous healthy-subject studies, is well tolerated, and was well below the dose in the US prescribing information. A dose of $20 \mathrm{mg}$ omeprazole daily is within the recommended dose as prescribed in the US labeling [11]. In addition, multiple doses for 5 consecutive days ensured maximum inhibition of acid secretion by omeprazole, as this pharmacodynamic response reaches a plateau after 4 days of dosing.

\section{Study Procedures}

On day 1 of period 1 , subjects were randomized to one of two treatment sequences: $\mathrm{ABC}$ or BAC.

On day 1 of periods 1 and 2, a single oral dose of cysteamine bitartrate DR was administered with orange juice (treatment $\mathrm{A}$ ) or water (treatment B), followed by blood sampling for $24 \mathrm{~h}$ for the determination of plasma cysteamine levels.

In period 3, multiple oral doses of omeprazole with water were administered once daily for 5 consecutive days (days 1-5), co-administered with a single oral dose of cysteamine bitartrate DR with water (treatment C) on day 5. Blood samples for the determination of plasma cysteamine levels were taken for $24 \mathrm{~h}$ following cysteamine bitartrate DR dosing on Day 5.

There was a washout period of 2 days between the first dosing in each period. Subjects were followed up approximately 14 days after the last study drug administration to determine whether any AE had occurred since the last dose of study drug(s). 


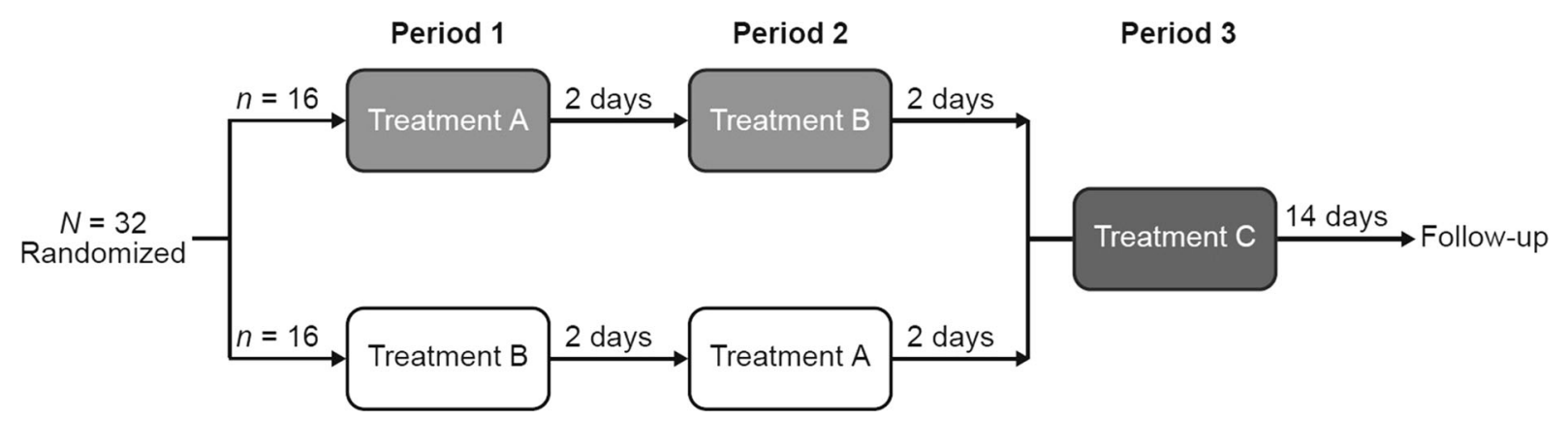

Fig. 1 Study design. Treatment $A$ single dose of cysteamine bitartrate delayed-release capsules $(600 \mathrm{mg})$ with orange juice on day 1 . Treatment $B$ single dose of cysteamine bitartrate delayed-release capsules $(600 \mathrm{mg})$ with water on day 1. Treatment $C$ omeprazole $(20 \mathrm{mg})$ with water once

\section{Outcome Measures}

The following primary PK parameters were assessed: area under the concentration-time curve from time 0 to the time of the last observed/measured non-zero concentration $\left(\mathrm{AUC}_{0-t}\right)$, area under the concentration-time curve from time 0 extrapolated to infinity $\left(\mathrm{AUC}_{0-\infty}\right)$, maximum observed concentration $\left(C_{\max }\right)$, and time to reach $C_{\max }\left(t_{\max }\right)$. In addition, the percent of $\mathrm{AUC}_{0-\infty}$ extrapolated (AUC\%extrap), apparent first-order terminal elimination rate constant $\left(k_{\mathrm{el}}\right)$, apparent firstorder terminal elimination half-life $\left(t_{1 / 2}\right)$, apparent total plasma clearance after extravascular administration (CL/F), and the apparent volume of distribution during the terminal elimination phase after extravascular administration $\left(V_{\mathrm{z}} / F\right)$ were calculated. All PK parameters were calculated using Phoenix $^{\circledR}$ WinNonlin $^{\circledR}$, v.6.3 from the 24-h plasma cysteamine concentration-time data.

Blood samples for determining plasma cysteamine concentrations were obtained at the following time points: pre-dose $(0 \mathrm{~h}), 0.5,1,2$, $2.5,3,3.5,4,6,8,10,12,18$, and 24 h postdose.

Safety was assessed via evaluation of AEs classified by the Medical Dictionary for Regulatory Activities (MedDRA) v.19.0, physical examination, 12-lead electrocardiograms, vital signs (including systolic and diastolic blood pressure, pulse rate, temperature, and respiratory rate), daily for 5 days + single dose of cysteamine bitartrate delayed-release capsules $(600 \mathrm{mg})$ with water on day 5 . On each of the 5 days, omeprazole was administered within $\pm 1 \mathrm{~h}$ of the dosing time on day 1

body weight, and clinical laboratory tests. Only treatment-emergent AEs (TEAEs) were summarized.

\section{Statistical Analysis}

A sample size of 28 subjects was calculated using a power of at least $80 \%$ and an alpha error of $5 \%$. The power was defined as the probability of having a $90 \%$ confidence interval (CI) to detect a ratio within the acceptance criteria of $80.00-125.00 \%$. A true ratio between $95 \%$ and $105 \%$ was assumed, and an intra-subject coefficient of variation $(\mathrm{CV})$ of $\sim 25 \%$ obtained from previous data was used. A total of 32 subjects were dosed to allow for possible dropouts.

Summary statistics were calculated for all nominal concentration-time points and for plasma cysteamine PK parameters. In addition, geometric means and geometric CVs were calculated for $C_{\max }$ and AUC parameters.

For PK parameters, analysis of variance was performed on the log-transformed $\mathrm{AUC}_{0-t}$, $\mathrm{AUC}_{0-\infty}$, and $C_{\max }$ with sequence and treatment as fixed effects and subject nested within sequence as a random effect. Period was not considered in the statistical model because the third treatment period was confounded with treatment $\mathrm{C}$. The linear model included calculation of least-squares means (LSM), the difference between treatment LSM, and the standard error associated with this difference. Ratios of LSM were calculated using the exponentiation 
of the difference between treatment LSM from the analyses on the log-transformed $\mathrm{AUC}_{0-t}$, $\mathrm{AUC}_{0-\infty}$, and $C_{\text {max }}$. These ratios were expressed as a percentage relative to the appropriate reference treatment (treatment A or treatment B), depending on the comparison of interest. Consistent with the two one-sided tests, 90\% CIs for the ratios were derived by exponentiation of the CIs obtained for the difference between treatment LSM resulting from the analyses on the log-transformed $\mathrm{AUC}_{0-t}$, $\mathrm{AUC}_{0-\infty}$, and $C_{\max }$, and were expressed as a percentage relative to treatment A or treatment $\mathrm{B}$, depending on the following comparisons of interest:

- Cysteamine bitartrate DR capsules + water (treatment B) compared with cysteamine bitartrate DR capsules + orange juice (treatment A)

- Omeprazole + cysteamine bitartrate DR capsules + water (treatment C) compared with cysteamine bitartrate DR capsules + water (treatment B)

- Omeprazole + cysteamine bitartrate DR capsules + water (treatment C) compared with cysteamine bitartrate DR capsules + orange juice (treatment $\mathrm{A}$ ).

Nonparametric analysis (Wilcoxon signed rank test) was performed on $t_{\max }$, which included calculation of median and 95\% CIs of the differences among treatments.

All plasma cysteamine concentrations and/ or PK parameter descriptive statistics were generated using SAS $^{\circledR}$, v.9.3 (Cary, NC, USA).

\section{RESULTS}

\section{Subject Disposition and Demographics}

A total of 32 subjects (mean age, 41.7 years [range, 22-54 years], mean \pm SD BMI $27.6 \pm$ $2.6 \mathrm{~kg} / \mathrm{m}^{2}$ ) were randomly assigned equally to treatment sequence 1 (ABC, $n=16)$ or treatment sequence 2 (BAC, $n=16$ ), and all subjects completed the study. Baseline characteristics of the overall population and the two treatment sequence populations were similar (Table 1 ).

\section{Plasma Cysteamine Concentrations}

Linear mean plasma cysteamine concentration-time profiles for treatments $\mathrm{A}, \mathrm{B}$, and $\mathrm{C}$ are shown in Fig. 2. The peak mean plasma cysteamine concentration following coaministration of cysteamine bitartrate DR capsules with orange juice (treatment A, $1892 \mathrm{ng} /$ $\mathrm{mL}$ ) was higher compared with co-administration with water (treatment B, $1663 \mathrm{ng} / \mathrm{mL}$ ) or co-administration with omeprazole $20 \mathrm{mg}$ and water (treatment C, $1712 \mathrm{ng} / \mathrm{mL}$ ). Mean times to peak plasma cysteamine concentration were 3.5 , 3.0, and $2.5 \mathrm{~h}$ post-dose for treatments $\mathrm{A}, \mathrm{B}$, and $\mathrm{C}$, respectively (Fig. 2).

\section{Cysteamine Pharmacokinetics}

The $C_{\max }$ and AUC parameter values indicate similar peak and total exposures to cysteamine when cysteamine bitartrate was administered with water or orange juice, but exposures were slightly higher with co-administration of omeprazole (Table 2). Co-administration with omeprazole and water led to an earlier median $t_{\max }(2.50 \mathrm{~h})$ compared with water alone $(3.00 \mathrm{~h})$ or orange juice alone $(3.50 \mathrm{~h}$; Table 2$)$.

However, other PK parameters, including $t_{1 / 2}$, $\mathrm{CL} / F$, and $V_{\mathrm{z}} / F$, were similar for treatment groups $\mathrm{A}, \mathrm{B}$, and $\mathrm{C}$. The ratios of $\mathrm{AUC}$ and $C_{\max }$ between treatment sequences demonstrate that overall exposure to cysteamine was similar when it was administered with either water or orange juice, and when it was administered with omeprazole and either water or orange juice (Table 3). Furthermore, for all comparisons, ratios and 90\% CIs were within the $80-125 \%$ bioequivalence range for $\mathrm{AUC}_{0-t}$, $\mathrm{AUC}_{0-\infty}$, and $C_{\text {max }}$. However, $t_{\max }$ occurred significantly earlier when cysteamine was administered with water than when it was administered with orange juice (B-A difference $-0.50,95 \%$ CI -1.00 to $0.00, P=0.0425$ ), earlier with omeprazole than with water only (C-B difference $-0.50,95 \% \mathrm{CI}-1.00$ to -0.24 , $P=0.0017)$, and earlier with omeprazole than with orange juice only (C-A difference -1.00 , $95 \% \mathrm{CI}-1.47$ to $-0.75, P<0.0001)$. 
Table 1 Baseline demographic characteristics

\begin{tabular}{|c|c|c|c|}
\hline Characteristic & $\begin{array}{l}\text { Sequence } 1(\mathrm{ABC}) \\
n=16\end{array}$ & $\begin{array}{l}\text { Sequence } 2(\mathrm{BAC}) \\
n=16\end{array}$ & $\begin{array}{l}\text { Overall } \\
n=32\end{array}$ \\
\hline \multicolumn{4}{|l|}{ Sex, $n(\%)$} \\
\hline Female & $4(25)$ & $5(31)$ & $9(28)$ \\
\hline Male & $12(75)$ & $11(69)$ & $23(72)$ \\
\hline \multicolumn{4}{|l|}{ Race, $n(\%)$} \\
\hline American Indian or Alaskan native & 0 & $1(6)$ & $1(3)$ \\
\hline Black or African American & $2(13)$ & $1(6)$ & $3(9)$ \\
\hline White & $14(88)$ & $14(88)$ & $28(88)$ \\
\hline \multicolumn{4}{|l|}{ Age, years } \\
\hline Mean (SD) & $41.1(9.9)$ & $42.3(6.4)$ & $41.7(8.3)$ \\
\hline Median (min-max) & $41.5(22-54)$ & $44.0(30-52)$ & $43.5(22-54)$ \\
\hline \multicolumn{4}{|l|}{ BMI, $\mathrm{kg} / \mathrm{m}^{2}$} \\
\hline Mean (SD) & $27.8(2.5)$ & $27.4(2.8)$ & $27.6(2.6)$ \\
\hline Median (min-max) & $28.7(22.2-30.9)$ & $27.2(20.6-31.6)$ & $27.7(20.6-31.6)$ \\
\hline
\end{tabular}

$B M I$ body mass index, max maximum, min minimum

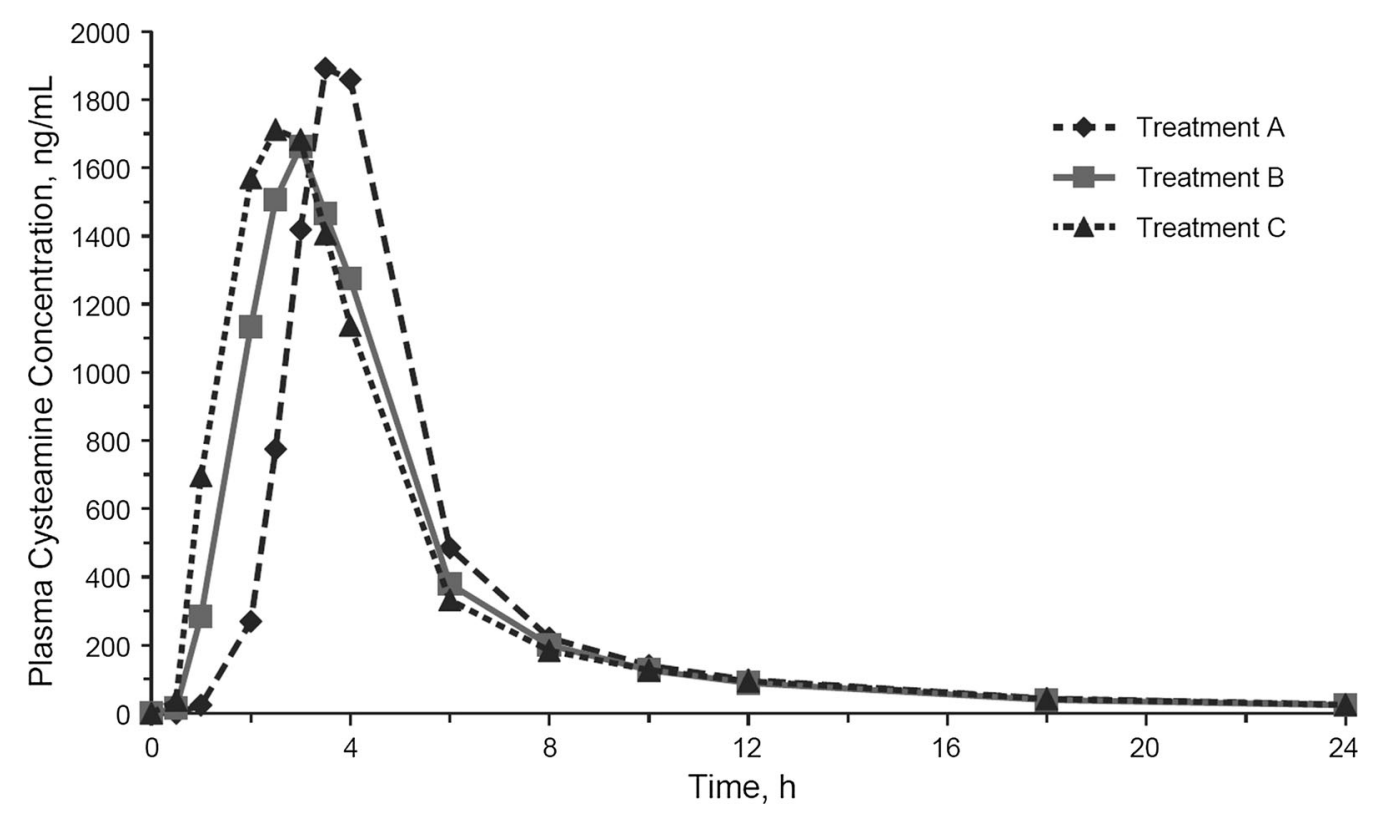

Fig. 2 Mean plasma cysteamine concentration-time profiles. Treatment $A$ single dose of cysteamine bitartrate delayed-release capsules $(600 \mathrm{mg})$ with orange juice. Treatment $B$ single dose of cysteamine bitartrate delayed- release capsules $(600 \mathrm{mg})$ with water. Treatment $C$ omeprazole $(20 \mathrm{mg})$ with water once daily for 5 days + single dose of cysteamine bitartrate delayed-release capsules $(600 \mathrm{mg})$ with water on day 5 
Table 2 Pharmacokinetics of cysteamine according to treatment group

\begin{tabular}{llll}
\hline Parameter $^{\mathbf{a}}$ & $\begin{array}{l}\text { Treatment A } \\
\boldsymbol{n}=\mathbf{3 2}\end{array}$ & $\begin{array}{l}\text { Treatment B } \\
\boldsymbol{n}=\mathbf{3 2}\end{array}$ & $\begin{array}{l}\text { Treatment C } \\
\boldsymbol{n}=\mathbf{3 2}\end{array}$ \\
\hline $\mathrm{AUC}_{0-t}(\mathrm{ng} \cdot \mathrm{h} / \mathrm{mL})^{\mathrm{a}}$ & $6724.8(29.0)$ & $6788.4(28.7)$ & $7313.0(25.8)$ \\
$\mathrm{AUC}_{0-\infty}(\mathrm{ng} \cdot \mathrm{h} / \mathrm{mL})^{\mathrm{a}}$ & $6936.3(28.9)$ & $6983.4(29.0)$ & $7522.9(26.0)$ \\
$C_{\max }(\mathrm{ng} / \mathrm{mL})^{\mathrm{a}}$ & $2169(35.0)$ & $2010(39.9)$ & $2223(30.4)$ \\
$t_{\max }(\mathrm{h})^{\mathrm{b}}$ & $3.50(2.5-6.0)$ & $3.00(2.0-6.0)$ & $2.50(1.0-4.1)$ \\
$t_{1 / 2}(\mathrm{~h})^{\mathrm{c}}$ & $5.79 \pm 0.80$ & $5.95 \pm 0.78$ & $5.99 \pm 0.50$ \\
$\mathrm{CL} / \mathrm{F}(\mathrm{L} / \mathrm{h})^{\mathrm{c}}$ & $89.9 \pm 25.2$ & $89.3 \pm 24.8$ & $82.4 \pm 21.7$ \\
$\mathrm{~V}_{\mathrm{z}} / \mathrm{F}(\mathrm{L})^{\mathrm{c}}$ & $746.9 \pm 218.9$ & $751.7 \pm 178.5$ & $707.6 \pm 183.4$ \\
\hline
\end{tabular}

$A U C_{0-t}$ area under the concentration-time curve from time 0 to the time of the last observed/measured non-zero concentration, $A U C_{0-\infty}$ area under the concentration-time curve from time 0 extrapolated to infinity, $C L / F$ apparent clearance, $C_{\max }$ maximum observed concentration, $C V$ coefficient of variation, max maximum, min minimum, $P K$ pharmacokinetic, $t_{1 / 2}$ half-life, $t_{\max }$ time to maximum concentration, $V_{z} / F$ apparent volume of distribution

${ }^{a}$ Geometric mean (geometric \%CV)

b Median (min-max)

${ }^{c}$ Mean \pm SD

\section{Safety}

Overall, no deaths, serious AEs, discontinuations due to AEs, or clinically significant changes in vital signs, clinical laboratory results, or electrocardiography occurred in any subjects. In total, 20 TEAEs were reported by nine (28\%) subjects across all treatment periods, and the most common TEAE was diarrhea (five events in four subjects overall; Table 4). In total, ten TEAEs were considered related to cysteamine bitartrate DR, three events related to omeprazole, and two events related to both cysteamine bitartrate DR and omeprazole. All TEAEs were of mild severity, with the exception of one moderate severity headache, which was considered to be related to omeprazole.

\section{DISCUSSION}

In this study, peak and overall exposures to cysteamine were shown to be statistically equivalent when cysteamine bitartrate DR capsules were administered with orange juice, water, or with omeprazole and water at steady state for cysteamine bitartrate. Further, ratios of key PK parameters were entirely within the
80-125\% acceptance range for bioequivalence for all comparisons. The $t_{\max }$ occurred earlier when cysteamine bitartrate DR capsules were co-administered with omeprazole and water compared with treatment with cysteamine bitartrate DR capsules only (either water or orange juice). All treatments were well tolerated, and the study revealed no additional safety issues.

Suboptimal adherence is a known issue with cysteamine bitartrate formulations. This tendency relates, in part, to the high frequency of dosing, especially with the immediate-release formulation, which requires administration every $6 \mathrm{~h}$ [2]. Cysteamine bitartrate DR capsules, which allow for less frequent dosing, were formulated to release active drug at a $\mathrm{pH}$ of 5.5-6.0 in the small intestine $[12,13]$. Studies to date have shown that the PK of both immediate release and DR formulations of cysteamine bitartrate are adversely affected by concomitant food intake. In the case of DR capsules, a highfat meal substantially reduced exposure (as shown by AUC and $C_{\max }$ ) and doubled $t_{\max }$ compared with fasting administration [10]. This has justified the recommendation that cysteamine bitartrate be administered in the fasting state and at least $30 \mathrm{~min}$ before a 
Table 3 Cysteamine pharmacokinetics: statistical comparisons among treatment groups

\begin{tabular}{|c|c|c|c|c|}
\hline \multicolumn{5}{|c|}{ Water $(B)$ versus orange juice $(A)$} \\
\hline Parameter & $\begin{array}{l}\text { Treatment B } \\
n=32\end{array}$ & $\begin{array}{l}\text { Treatment A } \\
n=32\end{array}$ & B/A (\%) & $90 \% \mathrm{CI}$ \\
\hline $\mathrm{AUC}_{0-t}(\mathrm{ng} \cdot \mathrm{h} / \mathrm{mL})$ & 6788.4 & 6724.8 & 100.95 & $95.89-106.27$ \\
\hline $\mathrm{AUC}_{0-\infty}(\mathrm{ng} \cdot \mathrm{h} / \mathrm{mL})$ & 6983.4 & 6936.3 & 100.68 & $95.74-105.88$ \\
\hline$C_{\max }(\mathrm{ng} / \mathrm{mL})$ & 2010 & 2169 & 92.65 & $83.82-102.41$ \\
\hline \multicolumn{5}{|c|}{ Omeprazole $(C)$ versus water only $(B)$} \\
\hline Parameter & $\begin{array}{l}\text { Treatment C } \\
n=32\end{array}$ & $\begin{array}{l}\text { Treatment B } \\
n=32\end{array}$ & C/B (\%) & $90 \% \mathrm{CI}$ \\
\hline $\mathrm{AUC}_{0-t}(\mathrm{ng} \cdot \mathrm{h} / \mathrm{mL})$ & 7313.0 & 6788.4 & 107.73 & $102.33-113.41$ \\
\hline $\mathrm{AUC}_{0-\infty}(\mathrm{ng} \cdot \mathrm{h} / \mathrm{mL})$ & 7522.9 & 6983.4 & 107.72 & $102.44-113.29$ \\
\hline$C_{\max }(\mathrm{ng} / \mathrm{mL})$ & 2223 & 2010 & 110.60 & $100.06-122.26$ \\
\hline \multicolumn{5}{|c|}{ Omeprazole $(\mathrm{C})$ versus orange juice only $(\mathrm{A})$} \\
\hline Parameter & $\begin{array}{l}\text { Treatment C } \\
n=32\end{array}$ & $\begin{array}{l}\text { Treatment A } \\
n=32\end{array}$ & C/A (\%) & $90 \% \mathrm{CI}$ \\
\hline $\mathrm{AUC}_{0-t}(\mathrm{ng} \cdot \mathrm{h} / \mathrm{mL})$ & 7313.0 & 6724.8 & 108.75 & $103.30-114.48$ \\
\hline $\mathrm{AUC}_{0-\infty}(\mathrm{ng} \cdot \mathrm{h} / \mathrm{mL})$ & 7522.9 & 6936.3 & 108.46 & $103.13-114.06$ \\
\hline$C_{\max }(\mathrm{ng} / \mathrm{mL})$ & 2223 & 2169 & 102.47 & $92.70-113.27$ \\
\hline
\end{tabular}

$A U C_{0-t}$ area under the concentration-time curve from time 0 to the time of the last observed/measured non-zero concentration, $A U C_{0-\infty}$ area under the concentration-time curve from time 0 extrapolated to infinity, $C I$ confidence interval, $C_{\max }$ maximum observed concentration

carbohydrate-rich meal [4]. In addition, it has been recommended that cysteamine bitartrate be administered with juice (but not grapefruit juice). Although the results of this study do not substantially alter these recommendations, they do demonstrate that co-administration with water did not adversely affect the bioavailability of cysteamine bitartrate DR. Further, co-administration with omeprazole also did not significantly reduce the overall exposure to cysteamine bitartrate, although the $t_{\max }$ was reduced significantly compared with administration with water or orange juice only.

The main strengths of this study are the use of randomization and a three-period treatment protocol, with washout periods between each treatment period. The main limitation of this study was the recruitment of healthy adult subjects rather than children with cystinosis, the main demographic group in clinical practice. The sample size was relatively small, but did meet the statistical test for evaluating bioequivalence. The study evaluated the PK of cysteamine in the presence of just one of the available PPIs, but we do not consider this a limitation. Maximal inhibition of acid secretion was achieved with omeprazole $20 \mathrm{mg}$, so the results would not have differed if additional PPIs had been tested.

\section{CONCLUSIONS}

Mean plasma peak and total exposures of cysteamine were similar when cysteamine bitartrate DR capsules were administered with 
Table 4 Subjects with treatment-emergent adverse events by treatment group

\begin{tabular}{lllllll}
\hline TEAEs, $\boldsymbol{n}$ (\%) & $\begin{array}{l}\text { Treatment A A } \\
n=32\end{array}$ & $\begin{array}{l}\text { Treatment B } \\
n=32\end{array}$ & $\begin{array}{l}\text { Treatment C } \\
\text { Days 1-4 } \\
n=32\end{array}$ & $\begin{array}{l}\text { Treatment C } \\
\text { Days 5 }+ \\
n=32\end{array}$ & $\begin{array}{l}\text { Treatment C } \\
\text { Overall } \\
\boldsymbol{n}=\mathbf{3 2}\end{array}$ & $\begin{array}{l}\text { Total } \\
n=32\end{array}$ \\
\hline Subjects with TEAEs (\% total) & $7(22)$ & $2(6)$ & $3(9)$ & $2(6)$ & $4(13)$ & $9(28)$ \\
Abdominal pain & 0 & $1(3)$ & 0 & 0 & 0 & $1(3)$ \\
Constipation & $1(3)$ & 0 & 0 & 0 & 0 & $1(3)$ \\
Diarrhea & $4(13)$ & $1(3)$ & 0 & 0 & 0 & $4(13)$ \\
Epigastric discomfort & $1(3)$ & 0 & 0 & 0 & 0 & $1(3)$ \\
Upper respiratory tract infection & $1(3)$ & 0 & 0 & 0 & 0 & $1(3)$ \\
Limb discomfort & 0 & $1(3)$ & 0 & 0 & 0 & $1(3)$ \\
Dizziness & $1(3)$ & 0 & $1(3)$ & 0 & $1(3)$ & $2(6)$ \\
Dysgeusia & 0 & 0 & 0 & $1(3)$ & $1(3)$ & $1(3)$ \\
Head discomfort & 0 & 0 & 0 & $1(3)$ & $1(3)$ & $1(3)$ \\
Headache & 0 & 0 & $1(3)$ & 0 & $1(3)$ & $1(3)$ \\
Presyncope & $1(3)$ & 0 & 0 & 0 & $1(3)$ & $1(3)$ \\
Micturition disorder & 0 & 0 & $1(3)$ & 0 & $1(3)$ & $1(3)$ \\
Dysmenorrhea & 0 & $1(3)$ & 0 & 0 & 0 & $1(3)$ \\
Throat tightness & 0 & 0 & 0 & $1(3)$ & $1(3)$ & $1(3)$ \\
Dry skin & 0 & $1(3)$ & 0 & 0 & 0 & $1(3)$ \\
\hline
\end{tabular}

$T E A E$ treatment-emergent adverse event

water or orange juice, as well as with and without omeprazole $20 \mathrm{mg}$ once daily for 5 days. The median $t_{\max }$ was $0.5 \mathrm{~h}$ later when cysteamine bitartrate DR capsules were administered with orange juice compared with water and earlier by $0.5 \mathrm{~h}$ when administered with omeprazole. However, PK profiles demonstrating exposure were essentially similar for all administration methods.

\section{ACKNOWLEDGEMENTS}

Funding. Sponsorship for this study and article processing charges was funded by Horizon Pharma, Inc., which was involved in the study design, data collection, data analysis, and preparation of the manuscript. Celerion also participated in the study design, data collection, data analysis, and preparation of the manuscript. All authors had full access to all of the data in this study and take complete responsibility for the integrity of the data and accuracy of the data analysis. Open access fees for this publication were funded by Horizon Pharma, Inc.

Medical Writing Assistance. Medical writing assistance was provided by Mark Snape, MB BS, CMPP, of Excel Scientific Solutions, and was funded by Horizon Pharma, Inc. Excel's services complied with Good Publication Practice 3 (GPP3) guidelines.

Authorship. All named authors meet the International Committee of Medical Journal 
Editors (ICMJE) criteria for authorship for this manuscript, take responsibility for the integrity of the work as a whole, and have given final approval to the version to be published. All authors had full access to all of the data in this study and take complete responsibility for the integrity of the data and accuracy of the data analysis.

Author Contributions. Danielle Armas, Mohammad Obaidi, Yuli Xie, and Meg Brannagan participated in study design and data acquisition and analysis. Nils F. Confer and Gregg C. Checani participated in study design and data analysis. Robert J. Holt participated in data analysis. All authors participated in the interpretation of study results and in the drafting, critical revision, and approval of the final version of the manuscript.

Other Contributors. The authors would like to thank all study participants and the staff at Celerion for the execution of this study.

Disclosures. Danielle Armas is an employee of Celerion Inc., which provided contract research services for the study sponsor. Mohammad Obaidi is an employee of Celerion Inc., which provided contract research services for the study sponsor. Yuli Xie is an employee of Celerion Inc., which provided contract research services for the study sponsor. Meg Brannagan is an employee of Celerion Inc., which provided contract research services for the study sponsor. Robert J. Holt is an employee of Horizon Pharma, Inc., which sponsored the study. Nils F. Confer is an employee of Horizon Pharma, Inc., which sponsored the study. Gregg C. Checani is an employee of Horizon Pharma, Inc., which sponsored the study.

Compliance with Ethics Guidelines. Approval to conduct this study was granted by the institutional review board at the study site (Chesapeake Research Review). All procedures followed were in accordance with the ethical standards of the responsible committee on human experimentation (institutional and national) and with the Helsinki Declaration of 1964, as revised in 2013. Written informed consent was obtained from all patients for being included in the study.

Data Availability. The datasets generated during and/or analyzed during the current study are not publicly available because they are not within the scope of FDAAA Section 801, but they are available from the corresponding author on reasonable request.

Open Access. This article is distributed under the terms of the Creative Commons Attribution-NonCommercial 4.0 International License (http://creativecommons.org/licenses/ by-nc/4.0/), which permits any noncommercial use, distribution, and reproduction in any medium, provided you give appropriate credit to the original author(s) and the source, provide a link to the Creative Commons license, and indicate if changes were made.

\section{REFERENCES}

1. Elmonem MA, Veys KR, Soliman NA, et al. Cystinosis: a review. Orphanet J Rare Dis. 2016;11:47.

2. Emma F, Nesterova G, Langman C, et al. Nephropathic cystinosis: an international consensus document. Nephrol Dial Transplant. 2014;29(Suppl 4):iv87-94.

3. Mylan Pharmaceuticals. CYSTAGON ${ }^{\circledR}$ (cysteamine bitartrate) capsules: prescribing information. 2007. https://dailymed.nlm.nih.gov/dailymed/drugInfo. cfm?setid=f495b76d-96c6-48e5-8fa330a4336628eb. Accessed 7 November 2017.

4. Horizon Pharma. PROCYSBI ${ }^{\circledR}$ (cysteamine bitartrate) delayed-release capsules: prescribing information. Revised March 2016 http://www. horizonpharma.com/medicines/rare-diseases/ procysbi/. Accessed 7 November 2017.

5. Kleta R, Bernardini I, Ueda M, et al. Long-term follow-up of well-treated nephropathic cystinosis patients. J Pediatr. 2004;145(4):555-60.

6. Kleta R, Gahl WA. Pharmacological treatment of nephropathic cystinosis with cysteamine. Expert Opin Pharmacother. 2004;5(11):2255-62.

7. Markello TC, Bernardini IM, Gahl WA. Improved renal function in children with cystinosis treated 
with cysteamine. N Engl J Med. 1993;328(16): 1157-62.

8. Dohil R, Cabrera BL. Treatment of cystinosis with delayed-release cysteamine: 6-year follow-up. Pediatr Nephrol. 2013;28(3):507-10.

9. Langman CB, Greenbaum LA, Sarwal M, et al. A randomized controlled crossover trial with delayedrelease cysteamine bitartrate in nephropathic cystinosis: effectiveness on white blood cell cystine levels and comparison of safety. Clin J Am Soc Nephrol. 2012;7(7):1112-20.

10. Dohil R, Rioux P. Pharmacokinetic studies of cysteamine bitartrate delayed-release. Clin Pharmacol Drug Dev. 2013;2(2):178-85.
11. AstraZeneca Pharmaceuticals LP. PRILOSEC ${ }^{\circledR}$ (omeprazole) delayed-release capsules: prescribing information. Updated October 2016. https:// dailymed.nlm.nih.gov/dailymed/drugInfo.cfm?se tid=A1B077E6-B070-43F2-A98E-380CC635419D. Accessed 7 November 2017.

12. Dohil R, Fidler M, Gangoiti JA, et al. Twice-daily cysteamine bitartrate therapy for children with cystinosis. J Pediatr. 2010;156(1):71-5 (e1-3).

13. Dohil R, Gangoiti JA, Cabrera BL, et al. Long-term treatment of cystinosis in children with twice-daily cysteamine. J Pediatr. 2010;156(5):823-7. 